\title{
LOGARITHMIC COMPARISON THEOREM VERSUS GAUSS-MANIN SYSTEM FOR ISOLATED SINGULARITIES
}

\author{
MATHIAS SCHULZE
}

\begin{abstract}
For quasihomogeneous isolated hypersurface singularities, the logarithmic comparison theorem has been characterized explicitly by Holland and Mond. In the nonquasihomogeneous case, we give a necessary condition for the logarithmic comparison theorem in terms of the Gauss-Manin system of the singularity. It shows in particular that the logarithmic comparison theorem can hold for a nonquasihomogeneous singularity only if 1 is an eigenvalue of the monodromy.
\end{abstract}

\section{INTRODUCTION}

Let $D$ be a hypersurface in a complex manifold $X$ with complement $j: U=$ $X \backslash D \hookrightarrow X$. Then Grothendieck's comparison theorem Gro66 states that the De Rham morphism

$$
\Omega_{X}^{\bullet}(* D) \rightarrow \mathbf{R} j_{*} \mathbb{C}_{U}
$$

is a quasi-isomorphism. In particular, for Stein $X$, each cohomology class $c \in$ $H^{k}(U ; \mathbb{C})$ is represented as $c(\sigma)=\int_{\sigma} \omega$ by a differential $k$-form $\omega$ with finite pole order along $D$. The natural question of limiting this pole order dates back to Griffiths Gri69] and has been studied later by Deligne and Dimca DD90, Dim91, Karpishpan [Kar91], and others.

For a normal crossing divisor $D=\left\{x_{1} \cdots x_{k}=0\right\} \subseteq \mathbb{C}^{n}=X$, the poles can be restricted to simple poles. More precisely, the inclusion

$$
\Omega^{\bullet}(\log D) \hookrightarrow \Omega_{X}^{\bullet}(* D)
$$

of the complex of logarithmic differential forms, generated by $\frac{d x_{1}}{x_{1}}, \ldots, \frac{d x_{k}}{x_{k}}, d x_{k+1}, \ldots, d x_{n}$, is a quasi-isomorphism. This fact plays a crucial role in Deligne's mixed Hodge theory [Del71, §3].

Saito Sai80] extended the definition of the complex $\Omega^{\bullet}(\log D)$ of logarithmic differential forms to general hypersurfaces $D$. In analogy with Grothendieck's theorem, one says that the logarithmic comparison theorem holds for $D$ if (1) is a quasi-isomorphism. The problem of characterizing such $D$ has been studied essentially in the extremal cases of isolated singularities and of free divisors. In both cases the complete solution is still missing.

The overlap of the two cases, the plane curve case, is completely understood: The logarithmic comparison theorem is equivalent to quasihomogeneity of the singularities CMMNMCJ02]. For free divisors, the normal crossing case has been

Date: August 31, 2021.

1991 Mathematics Subject Classification. 32S35, 32S40, 14F40.

Key words and phrases. isolated singularity, de Rham cohomology, Gauss-Manin system.

The author is grateful to Claus Hertling and to the anonymous referee for careful reading and useful suggestions, and to Michel Granger for helpful discussions. 
extended to the class of (weakly) locally quasihomogeneous free divisors, for which the logarithmic comparison theorem holds CJNMM96] ([NM08, Rem. 1.7.4]). For general free divisors, there is a D-module theoretic reformulation of the logarithmic comparison theorem based on a $\mathscr{D}_{X}(-\log D)$-duality analogous to the ordinary $\mathscr{D}_{X}$-duality CMNM05.

In the present note we are concerned with the case of isolated singularities. By the local nature of the problem, we can reduce to germs of spaces an maps:

$$
\begin{gathered}
X:=\left(\mathbb{C}^{n+1}, 0\right) \stackrel{f}{\longrightarrow}(\mathbb{C}, 0)=: T, \\
f \in \mathfrak{m}^{2} \subseteq \mathfrak{m}:=\mathfrak{m}_{X}=\langle x\rangle \subseteq \mathscr{O}:=\mathscr{O}_{X}=\mathbb{C}\{x\}, \quad \mathscr{O}_{T}=\mathbb{C}\{t\}, \\
D=\{x \mid f(x)=0\} \subseteq X, \quad U:=X \backslash D \stackrel{j}{\longrightarrow} X,
\end{gathered}
$$

where $f$ is a reduced equation of the isolated hypersurface singularity $D, x=$ $x_{0}, \ldots, x_{n}$ and $t$ are coordinates on $X$ and $T$. We shall tacitly identify $X$ with a Milnor representative Mil68. Note that the latter form a basis of Stein neighborhoods of $0 \in X$ and it suffices to check the logarithmic comparison theorem on global sections over such neighborhoods by CJNMM96, Lem. 2.5].

The main result for isolated singularities due to Holland and Mond HM98] covers the case of quasihomogeneous singularities.

Theorem 1 (Holland, Mond). Let $D=\{x \mid f(x)=0\} \subseteq X$ be a quasihomogeneous isolated hypersurface singularity of degree $r$ with respect to positive integer weights $w_{1}, \ldots, w_{n}$. Denote by $J_{f}$ the gradient ideal of $f$. Then the following conditions are equivalent:

(a) the logarithmic comparison theorem holds at 0 ;

(b) $\left(\mathbf{R}^{i} j_{*} \mathbb{C}_{U}\right)_{0}=0$ for $i \geq 2$;

(c) $\left(\mathscr{O}_{X, 0} / J_{f}\right)_{i r-\sum_{j} w_{j}}=0$ for $1 \leq i \leq n-1$;

(d) the link of 0 in $D$ is a $\mathbb{Q}$-homology sphere.

Furthermore, each of these statements implies that $\mathscr{H}^{i}\left(\Omega^{\bullet}(\log D)\right)=0$ for $i \geq 2$; for $n=2$ the reverse implication also holds.

For free divisors, it is conjectured, and proved for $n \leq 2$, that the logarithmic comparison theorem requires strong Euler homogeneity GS06. For isolated singularities, the latter property reduces to quasihomogeneity by Sai71 and one could expect that the logarithmic comparison theorem requires quasihomogeneity. Our main result confirms this expectation for a large class of isolated singularities defined by properties of the Gauss-Manin system $\mathscr{G}:=\int_{f}^{0} \mathscr{O}_{X}$. This is the direct image of the $\mathscr{D}_{X}$-module $\mathscr{O}_{X}$ along $f: X \rightarrow T$ and as such a $\mathscr{D}_{T}$-module.

Theorem 2. Let $D=\{x \mid f(x)=0\} \subseteq X$ be an isolated hypersurface singularity with Gauss-Manin system $\mathscr{G}=\int_{f}^{0} \mathscr{O}_{X}$, Brieskorn lattice $\mathscr{H}^{\prime \prime} \subseteq \mathscr{G}$, monodromy $M$, and spectrum $\alpha_{1}<\alpha_{2} \leq \cdots \leq \alpha_{n}$. Denote by $V$ the Kashiwara-Malgrange filtration on $\mathscr{G}$, by $C^{\alpha} \cong \operatorname{gr}_{V} \mathscr{G}$ the generalized $\alpha$-eigenspace of $t \partial_{t}$ in $\mathscr{G}$, by $H_{\alpha}$ the image of $\mathscr{H}^{\prime \prime} \cap V^{\alpha}$ in $C^{\alpha}$, and $N=\log \left(M_{u}\right): C^{\alpha} \rightarrow C^{\alpha}$ where $M_{u}$ is the unipotent part of $M$.

Under each of the following conditions the logarithmic comparison theorem can hold for $D$ only if $D$ is quasihomogeneous.

(a) 1 is not an eigenvalue of $M$; 
(b) $\alpha_{1}>0$

(c) $\alpha_{1}<0$ and $[u d x]_{0} \in H_{0} \oplus N\left(C^{0}\right)$ for some $u \in \mathscr{O}_{X}^{*}$;

(d) $\alpha_{1}<0=\alpha_{2}$ and $[d x]_{0} \in H_{0} \oplus N\left(C^{0}\right)$.

In the case $\alpha_{1}=0$, our approach does not give a statement. The methods developed in Sch02, Sch04b serve to check the conditions in Theorem 2 algorithmically. We have used the Singular GPS05 implementation Sch04a of these methods to compute the following example which is out of the scope of Theorem 1.

Example 3. Consider the isolated singularity $D$ defined by $f=x^{5}+x^{2} y^{2}+y^{5}+z^{5}$. By a Gröbner basis computation, one easily verifies that $f \notin\left\langle\frac{\partial f}{\partial x}, \frac{\partial f}{\partial z}, \frac{\partial f}{\partial z}\right\rangle$ which shows $D$ is not quasihomogeneous. The spectrum of $f$ consists of the collection of $\alpha \in \mathbb{Q}$ with multiplicity $\mu_{\alpha} \in \mathbb{N}$ listed in Table 1 As there are no integer spectral numbers, the monodromy does not have an eigenvalue 1 . Thus, Theorem 2 implies that the logarithmic comparison theorem does not hold for $D$.

Table 1: Spectrum of $f=x^{5}+x^{2} y^{2}+y^{5}+z^{5}$

\begin{tabular}{c|c|c|c|c|c|c|c|c|c|c|c|c|c}
$\alpha$ & $-\frac{3}{10}$ & $-\frac{1}{10}$ & $\frac{1}{10}$ & $\frac{1}{5}$ & $\frac{3}{10}$ & $\frac{2}{5}$ & $\frac{1}{2}$ & $\frac{3}{5}$ & $\frac{7}{10}$ & $\frac{4}{5}$ & $\frac{9}{10}$ & $\frac{11}{10}$ & $\frac{13}{10}$ \\
\hline$\mu_{\alpha}$ & 1 & 3 & 5 & 1 & 7 & 1 & 8 & 1 & 7 & 1 & 5 & 3 & 1
\end{tabular}

We shall prove Theorem 2 in Section 3 after some preparations on logarithmic vector fields in the following Section 2 .

\section{LOGARITHMIC VECTOR FIELDS}

We shall assume throughout that $D$ is an isolated singularity and use the notation in (2). Denote by

$$
\operatorname{Der}(-\log D):=\{\delta \mid \delta(f) \in \mathscr{O} f\} \subseteq \operatorname{Der}:=\operatorname{Der}_{\mathbb{C}}(\mathscr{O}) \cong \mathscr{O}^{n+1}
$$

the $\mathscr{O}$-module of logarithmic vector fields along $D$. We may assume that

$$
\operatorname{Der}(-\log D) \subseteq \mathfrak{m} \text { Der }=: \Delta
$$

which means that $D \not D^{\prime} \times \mathbb{C}$. Let $\delta_{0}$ be the image of $\delta \in \Delta$ and $\operatorname{Der}(-\log D)_{0}$ that of the infinite Lie algebra $\operatorname{Der}(-\log D)$ under the Lie algebra homomorphism

$$
\Delta \stackrel{\pi_{0}}{\longrightarrow} \Delta / \mathfrak{m} \Delta=: \Delta_{0} \cong \mathfrak{g l}_{n+1}(\mathbb{C}), \quad \sum_{i, j=1}^{n} a_{j, i}(x) x_{i} \partial_{j} \mapsto\left(a_{i, j}(0)\right)_{i, j},
$$

where we abbreviate $\partial_{i}:=\frac{\partial}{\partial x_{i}}$ for $i=0, \ldots, n$. Note that $\operatorname{Der}(-\log D)_{0}$ is a finite Lie algebra. The basis $x=x_{0}, \ldots, x_{n}$ of $\mathfrak{m}$ defines a section of the map $\pi_{0}$ in (3) by which we can consider $\Delta_{0}$ and $\operatorname{Der}(-\log D)_{0}$ as Lie subalgebras of $\Delta$. We call $\delta=\delta_{0} \in \Delta$ semisimple if the corresponding matrix $\pi_{0}(\delta)$ has this property. If $\tau_{0}(\delta)$ is a nilpotent matrix (but not necessarily $\delta=\delta_{0}$ ), we call $\delta \in \Delta$ nilpotent. While semisimplicity depends on the coordinate system, nilpotency is an intrinsic property. Any $\delta \in \Delta$ can be decomposed as

$$
\delta=\delta_{S}+\delta_{N}
$$

into a semisimple $\delta_{S}$ and nilpotent $\delta_{N}$. Note that $\delta_{S}$ is just the semisimple part of $\tau_{0}(\delta)$ in the linear algebra sense.

Proposition 4. If $D$ is of order at least 3 (which means that $f \in \mathfrak{m}^{3}$ ) but not quasihomogeneous then $\operatorname{Der}(-\log D)$ contains only nilpotent vector fields. 
Proof. We may replace $\operatorname{Der}(-\log D)$ by its m-adic completion $\widehat{\operatorname{Der}}(-\log D)=$ $\operatorname{Der}(-\log \widehat{D})$ where $\widehat{D}$ is defined by the same $f \in \mathscr{O} \subseteq \widehat{\mathscr{O}}$ considered as a formal power series. Indeed, $D$ has an isolated singularity if and only if $\widehat{D}$ has and quasihomogeneity is equivalent to Euler homogeneity by Sai71 which is invariant under completion. Moreover, $\widehat{\operatorname{Der}}(-\log D)_{0}=\operatorname{Der}(-\log D)_{0}$ and the notion of nilpotency is preserved.

Let $\widehat{\delta} \in \operatorname{Der}(-\log \widehat{D})$ and decompose it as in (4). By [GS06, Thm. 5.4], there is a (formal) coordinate system with respect to which $\sigma:=\widehat{\delta}_{S} \in \operatorname{Der}(-\log \widehat{D})$ and a defining equation $\widehat{f} \in \widehat{\mathscr{O}}$ of $\widehat{D}$ such that $\sigma(\widehat{f}) \in \mathbb{Q} \widehat{f}$. We must have $\sigma(\widehat{f})=0$ as otherwise $\widehat{D}$ would be quasihomogeneous by Sai71. Assume that $\sigma \neq 0$. This means that the monomial support of $\widehat{f}$ lies in a proper vector subspace.

As $\widehat{f}$ has an isolated critical point, [Sai71, Cor. 1.6] states that, for each $j=$ $0, \ldots, n$, there must be a monomial with exponent $m e_{j}$ or $m e_{j}+e_{j^{\prime}}$ in the monomial support of $\widehat{f}$. But, by the order hypothesis, $\widehat{f} \in \widehat{\mathfrak{m}}^{3}$ which implies that these monomials are linearly independent. This contradicts to the monomial support of $\widehat{f}$ having codimension at least one and finishes the proof.

Dropping the order hypothesis in Proposition 4, a weaker statement holds.

Proposition 5. If $D$ is not quasihomogeneous then $\operatorname{tr}\left(\delta_{0}\right)=0$ for all $\delta \in \operatorname{Der}(-\log D)$.

Proof. If $f \in \mathfrak{m}^{3}$ then we may assume by Proposition 4 that $\delta_{0}$ is a lower triangular matrix and the claim follows.

In the general case, we can assume by the Splitting Lemma that

$$
f(x)=f^{\prime}\left(x^{\prime}\right)+\sum_{i}\left(x_{i}^{\prime \prime}\right)^{2}, \quad x=\left(x^{\prime}, x^{\prime \prime}\right), \quad \partial=\left(\partial^{\prime}, \partial^{\prime \prime}\right), \quad f^{\prime} \in \mathfrak{m}^{3} .
$$

With $D$ also

$$
D^{\prime}=\left\{x^{\prime} \mid f^{\prime}\left(x^{\prime}\right)=0\right\} \subseteq X^{\prime}
$$

is a nonquasihomogeneous isolated singularity by Sai71. Writing $\delta=\sum_{i} g_{i} \partial_{i}$, we have to check that the monomial $x_{i}$ does not occur in $g_{i}$. By definition of $\operatorname{Der}(-\log D), \delta$ corresponds to a syzygy of

$$
\begin{gathered}
x_{i}^{\prime} \partial_{j}^{\prime}(f)=x_{i}^{\prime} \partial_{j}^{\prime}\left(f^{\prime}\right) \in \mathfrak{m}^{3}, \\
x_{i}^{\prime \prime} \partial_{j}^{\prime \prime}(f)=2 x_{i}^{\prime \prime} x_{j}^{\prime \prime} \in \mathfrak{m}^{2} \backslash \mathfrak{m}^{3}, \\
x_{i}^{\prime \prime} \partial_{j}^{\prime}(f)=x_{i}^{\prime \prime} \partial_{j}^{\prime}\left(f^{\prime}\right) \in \mathfrak{m}^{3}, \quad x_{i}^{\prime} \partial_{j}^{\prime \prime}(f)=2 x_{i}^{\prime} x_{j}^{\prime \prime} \in \mathfrak{m}^{2} \backslash \mathfrak{m}^{3}, \quad x_{i}^{\prime} f, x_{j}^{\prime \prime} f \in \mathfrak{m}^{3} .
\end{gathered}
$$

By Sai71, $f$ can not occur with a constant coefficient in (7) as $D$ is assumed not to be quasihomogeneous. We are concerned only with the constant coefficients of (5) and (6) for $i=j$. Those of (6) are obviously zero. Setting $x^{\prime \prime}=0$ yields a syzygy of (5) and $x_{i}^{\prime} f^{\prime}$ that induces an element of $\operatorname{Der}\left(-\log D^{\prime}\right)$. Thus, the constant coefficients of (5) are zero for $i=j$ by the first part of the proof.

Let $\Omega_{X}^{\bullet}$ be the complex of holomorphic differential forms on $X$ and denote the volume form by

$$
d x:=d x_{0} \wedge \cdots \wedge d x_{n} \in \Omega_{X}^{n+1} .
$$

The complex of logarithmic differential forms along $D$ was introduced in [Sai80] as

$$
\Omega^{\bullet}(\log D):=\Omega_{X}^{\bullet}(D) \cap d^{-1} \Omega_{X}^{\bullet}(D) \subseteq \Omega_{X}^{\bullet}(* D) .
$$


Corollary 6. If $D$ is not quasihomogeneous then $0 \neq\left[\frac{u d x}{f}\right] \in \mathscr{H}^{n+1}\left(\Omega^{\bullet}(\log D)\right)$ for any $u \in \mathscr{O}^{*}$.

Proof. The module $\Omega^{n}(\log D)$ is the image of the inner product

$$
\begin{array}{r}
\operatorname{Der}(-\log D) \times \Omega^{n+1}(\log D) \longrightarrow \Omega^{n}(\log D) \\
(\delta, \omega) \longmapsto \iota_{\delta}(\omega)
\end{array}
$$

defined in Sai80, Lem. 1.6.ii]. As $\Omega^{n+1}(\log D)=\mathscr{O}_{X}(D) d x$, we have $\Omega^{n}(\log D)=$ $\iota_{\operatorname{Der}(-\log D)} d x / f$. Let $\delta=\sum_{i} g_{i} \partial_{i} \in \operatorname{Der}(-\log D)$ and note that $\delta(f) \in \mathfrak{m} f$ by nonquasihomogeneity of $D$ and [Sai71]. Then we compute

$$
\begin{aligned}
f d\left(\iota_{\delta}(d x) / f\right) & =d \circ \iota_{\delta}(d x)-(d f / f) \wedge \iota_{\delta}(d x) \\
& =\mathrm{L}_{\delta}(d x)-(\delta(f) / f) d x \\
& =\sum_{i} \partial_{i}\left(g_{i}\right) d x-(\delta(f) / f) d x \equiv \operatorname{tr}\left(\delta_{0}\right) d x \quad \bmod \mathfrak{m} .
\end{aligned}
$$

By Proposition [5 this implies that $d \Omega^{n}(\log D) \subseteq \mathfrak{m} \Omega^{n+1}(\log D)=\mathfrak{m} \Omega_{X}^{n+1}(D)$ and the claim follows.

\section{Gauss-Manin SYSTEM}

We keep our general assumption that $D$ is an isolated singularity and continue to use the notation in (2). Corollary 6 leads us to study the necessary condition

$$
0 \neq\left[\frac{u d x}{f}\right] \in \mathscr{H}^{n+1}\left(\Omega_{X}^{\bullet}(* D)\right) \text { for all } u \in \mathscr{O}^{*}
$$

for the logarithmic comparison theorem to hold for nonquasihomogeneous $D$. We shall reformulate this condition in terms of the Gauss-Manin system of $f: X \rightarrow T$ using [Kar91, §1-2] as a starting point.

Let $M$ be the monodromy on the canonical Milnor fiber $X_{\infty}$ of $f$ [SS85, §5]. By construction of $X_{\infty}$ and Mil68,

$$
H^{k}\left(X_{\infty} ; \mathbb{C}\right) \cong H^{k}\left(X_{t} ; \mathbb{C}\right)=0, \quad \text { if } k \neq 0, n,
$$

where $X_{t}:=f^{-1}(t)$ and $t \in T^{*}:=T \backslash\{0\}$. Then the cohomological Wang sequence reads

$$
0 \longrightarrow H^{n}(U ; \mathbb{C}) \longrightarrow H^{n}\left(X_{\infty} ; \mathbb{C}\right) \stackrel{M-1}{\longrightarrow} H^{n}\left(X_{\infty} ; \mathbb{C}\right) \longrightarrow H^{n+1}(U ; \mathbb{C}) \longrightarrow 0 .
$$

Recall that the eigenvalues of $M$ on $H^{n}\left(X_{\infty} ; \mathbb{C}\right)$ are roots of unity by the monodromy theorem [Bri70, Satz 4]. Decompose $M=M_{s} M_{u}$ into semisimple and unipotent part and let $H^{k}\left(X_{\infty} ; \mathbb{C}\right)_{\rho}$ denote the generalized $\rho$-eigenspace of $M$. Then $M-1$ has the same kernel and cokernel on $H^{n}\left(X_{\infty} ; \mathbb{C}\right)$ as on $H^{n}\left(X_{\infty} ; \mathbb{C}\right)_{1}, M$ coincides with $M_{u}$ on $H^{n}\left(X_{\infty} ; \mathbb{C}\right)_{1}$, and $M_{u}-1$ has the same kernel and cokernel as $N:=\log M_{u}$ on $H^{n}\left(X_{\infty} ; \mathbb{C}\right)_{1}$. Thus, (11) leads to an exact sequence

$$
0 \rightarrow H^{n}(U ; \mathbb{C}) \rightarrow H^{n+1}\left(X_{\infty} ; \mathbb{C}\right)_{1} \stackrel{N}{\rightarrow} H^{n+1}\left(X_{\infty} ; \mathbb{C}\right)_{1} \rightarrow H^{n+1}(U ; \mathbb{C}) \rightarrow 0 .
$$

To see the D-module structure hidden in (12) requires a refined approach. Let $\Gamma$ be the graph of $f$ and consider the maps $i(x)=(x, 0), j(x)=(x, f(x))$, and 
$p(x, t)=t$ in the diagram

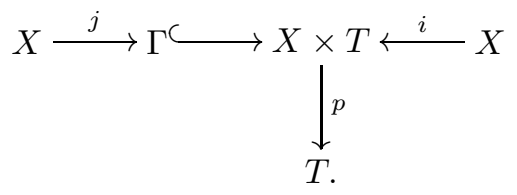

Then $i(X) \cap \Gamma=D, i^{*} \Omega_{X \times T / T}^{\bullet}(* \Gamma)=\Omega_{X}^{\bullet}(* D)$, and there is an exact sequence

$$
0 \longrightarrow \Omega_{X \times T / T}^{\bullet}(* \Gamma) \stackrel{t}{\longrightarrow} \Omega_{X \times T / T}^{\bullet}(* \Gamma) \longrightarrow i_{*} \Omega_{X}^{\bullet}(* D) \longrightarrow 0 .
$$

As $X$ is Stein and $\Omega_{X \times T / T}^{\bullet}$ consists of $\mathscr{O}_{X \times T^{-}}$-coherent and hence $p_{*}$-acyclic modules, the Poincaré Lemma shows that

$$
\mathbf{R}^{k} p_{*} \Omega_{X \times T / T}^{\bullet}=0, \quad \text { if } k \geq 1 .
$$

Therefore

$$
\begin{aligned}
\mathbf{R}^{k} p_{*}\left(\Omega_{X \times T / T}^{\bullet}(* \Gamma)\right) & =\mathbf{R}^{k} p_{*}\left(\Omega_{X \times T / T}^{\bullet}(* \Gamma) / \Omega_{X \times T / T}^{\bullet}\right) \\
& =\mathbf{R}^{k} f_{*} j^{-1}\left(\Omega_{X \times T / T}^{\bullet}(* \Gamma) / \Omega_{X \times T / T}^{\bullet}\right)=\int_{f}^{k-(n+1)} \mathscr{O}_{X}, \quad \text { if } k \geq 1,
\end{aligned}
$$

is the Gauss-Manin system of $f: X \rightarrow T$. As $\int_{f}^{k-n} \mathscr{O}_{X}$ is a $\mathscr{D}_{T}$-coherent regular extension of $\mathscr{O}_{T^{*}}\left(\bigcup_{t \in T^{*}} H^{k}\left(X_{t} ; \mathbb{C}\right)\right)$ to $T$, it follows from (10) that

$$
\int_{f}^{k} \mathscr{O}_{X}=0, \quad \text { if } k \neq 0,-n
$$

Using that $\mathbf{R} p_{*} i_{*}=\mathbf{R} p_{*} \mathbf{R} i_{*}=\mathbf{R}(p \circ i)_{*}=\mathbf{R} 0_{*}=\mathbf{R} \Gamma\left(X,,_{-}\right)=\mathbf{H}\left(X,{ }_{-}\right)$, Grothendieck's comparison theorem [Gro66] implies that

$$
\mathbf{R}^{k} p_{*} i_{*} \Omega_{X}^{\bullet}(* D)=\mathbf{H}^{k}\left(X, \Omega_{X}^{\bullet}(* D)\right)=h^{k}\left(\Gamma\left(X, \Omega_{X}^{\bullet}(* D)\right)\right)=\mathscr{H}^{k}\left(\Omega_{X}^{\bullet}(* D)\right) .
$$

So applying $\mathbf{R} p_{*}$ to (13) yields an exact sequence

$$
\begin{gathered}
0 \longrightarrow \mathscr{H}^{n}\left(\Omega_{X}^{\bullet}(* D)\right) \longrightarrow \mathscr{G} \stackrel{t}{\longrightarrow} \mathscr{G} \stackrel{\pi}{\longrightarrow} \mathscr{H}^{n+1}\left(\Omega_{X}^{\bullet}(* D)\right) \longrightarrow 0 \\
{[u d x] \longmapsto\left[\frac{u d x}{f}\right]}
\end{gathered}
$$

where $\mathscr{G}:=\int_{f}^{0} \mathscr{O}_{X}$.

By [Pha79, §15] and [SS85, Lem. 3.3], $\mathscr{G}$ can be represented in explicit form as

$$
\begin{gathered}
\mathscr{G} \cong \Omega_{X}^{n+1}[D] /(d-D \cdot d f \wedge) \Omega_{X}^{n}[D], \\
\partial_{t}\left[\frac{\omega}{(f-t)^{k}}\right]=\left[\frac{k ! \omega}{(f-t)^{k+1}}\right] \mapsto\left[\omega D^{k}\right] .
\end{gathered}
$$

The operator $\partial_{t}$ is invertible on $\mathscr{G}$ by $[$ Pha79, $\S 15.2 .2]$ and $\mathscr{G} \cong \mathbb{C}\left\{\left\{\partial_{t}^{-1}\right\}\right\}\left[\partial_{t}\right]^{\mu}$ where $\mu$ is the Milnor number of $f$ and $\mathbb{C}\left\{\left\{\partial_{t}^{-1}\right\}\right\}$ is the ring of microdifferential operators with constant coefficients. Composing the second map in (14) with $\partial_{t}^{-1}$, the operator $t$ in (14) can be replaced by $t \partial_{t}$ without changing the cokernel. This leads to an exact sequence

$$
0 \longrightarrow \mathscr{H}^{n}\left(\Omega_{X}^{\bullet}(* D)\right) \longrightarrow C^{0} \stackrel{t \partial_{t}}{\longrightarrow} C^{0} \stackrel{\pi}{\longrightarrow} \mathscr{H}^{n+1}\left(\Omega_{X}^{\bullet}(* D)\right) \longrightarrow 0
$$


where $C^{\alpha}$ denotes the generalized $\alpha$-eigenspace of the operator $t \partial_{t}$ on $\mathscr{G}$. The (decreasing) Kashiwara-Malgrange filtration $V^{\bullet}$ on $\mathscr{G}$ is essentially defined by $\operatorname{gr}_{V}^{\alpha} \mathscr{G} \cong C^{\alpha}$ and consists of free $\mathbb{C}\left\{\left\{\partial_{t}^{-1}\right\}\right\}$-modules of rank $\mu$. By [SS85, $\S 5$, p. 652], one can identify the vector spaces with endomorphisms

$$
\left(H^{n}\left(X_{\infty} ; \mathbb{C}\right)_{\lambda}, N\right) \cong\left(C^{\alpha},-2 \pi i\left(t \partial_{t}-\alpha\right)\right)
$$

We can thus identify $N=-2 \pi i t \partial_{t}$ in the sequences (12) and (16). By Gro66, also the outer terms of these sequences coincide.

With (9) and (14) in mind, we are interested in the image of the canonical map $\Omega_{X}^{n+1} \rightarrow \mathscr{G}($ see $(15)$ ), which is the Brieskorn lattice

$$
\mathscr{H}^{\prime \prime} \cong \Omega_{X}^{n+1} / d f \wedge d \Omega_{X}^{n-1}
$$

of $f$ [Bri70]. From (5) it follows easily that

$$
\mathscr{H}^{\prime \prime} / \partial_{t}^{-1} \mathscr{H}^{\prime \prime} \cong \Omega_{X}^{n+1} / d f \wedge \Omega_{X}^{n}=: \Omega_{f} \cong \mathbb{C}^{\mu} .
$$

By [Seb70], $\mathscr{H}^{\prime \prime}$ is a free $\mathbb{C}\{t\}$-module of rank $\mu$ and, by [Mal74, Lem. 4.5],

$$
\mathscr{H}^{\prime \prime} \subseteq V^{>-1}
$$

from which one can derive that $\mathscr{H}^{\prime \prime}$ is also a free $\mathbb{C}\left\{\left\{\partial_{t}^{-1}\right\}\right\}$-module of rank $\mu$ Pha77, Prop. 2.5].

For $g \in \mathscr{G}$ and $\alpha \in \mathbb{Q}$, we shall write $g_{\alpha}$ for the $C^{\alpha}$-component of $g$. The preceding arguments now show that (9) is equivalent to

$$
[u d x]_{0} \notin N\left(C^{0}\right) \text { for all } u \in \mathscr{O}^{*} .
$$

By (2), condition (a) in Theorem 2 implies that $C^{0}=0$ and the claim follows in that case. The spectrum of $f$ is defined as the spectrum $\alpha_{1} \leq \cdots \leq \alpha_{\mu}$ of the filtration induced by $V^{\bullet}$ on $\Omega_{f}$, that is,

$$
\#\left\{i \mid \alpha=\alpha_{i}\right\}=\operatorname{dim}_{\mathbb{C}} \operatorname{gr}_{V}^{\alpha} \Omega_{f} .
$$

Under condition (b) in Theorem 2, $\mathscr{H}^{\prime \prime} \subseteq V^{>0}$ and hence $[u d x]_{0}=0$ for all $u \in \mathscr{O}$. Thus, also in this case, Theorem (2) holds true.

In order to prove Theorem 2 under the assumption (다) or (d), let us assume that $\alpha_{1}<0$. From (18) and (19), we conclude that $C^{0} \cap \partial_{t}^{-1} \mathscr{H}^{\prime \prime}=0$ and hence

$$
C^{0} \subseteq V^{>-1} / \partial_{t}^{-1} \mathscr{H}^{\prime \prime} \supseteq \mathscr{H}^{\prime \prime} / \partial_{t}^{-1} \mathscr{H}^{\prime \prime} \cong \Omega_{f} .
$$

By [Sai91, Rem. 3.11], $\mathfrak{m} d x$ surjects onto $V^{>\alpha_{1}} \Omega_{f}$. In particular, $\alpha_{1}<\alpha_{2}$ and $[u d x] \in V^{\alpha_{1}} \backslash V^{>\alpha_{1}}$ for all $u \in \mathscr{O}^{*}$. Moreover, $\operatorname{gr}_{V}^{0} \mathscr{H}^{\prime \prime}=: H_{0} \subseteq C^{0}$ is in the image of $\mathfrak{m} d x$ by (21). By [SS85, Lem. 3.4 and $\S 6.5], \partial_{t}^{n-k} \mathscr{H}^{\prime \prime}$ induces the Hodge filtration $F^{k}$ on $\operatorname{gr}_{V}^{0} \mathscr{G}=C^{0}$ for which $N$ is a morphism of type -1 . Therefore, $H_{0}$ has a complement $G_{0}$ in $C^{0}$ such that $N\left(H_{0}\right) \subseteq G_{0}$. This shows that (20) is equivalent to

$$
[u d x]_{0} \notin H_{0} \oplus N\left(C^{0}\right) \text { for all } u \in \mathscr{O}^{*} .
$$

This proves Theorem 2 under the hypothesis (ㄷ).

Assume finally that $\alpha_{2}=0$. Then, modulo $\mathbb{C}^{*}$, the $G_{0}$-component of $[u d x]_{0}$ is independent of $u \in \mathscr{O}^{*}$ and (22) is equivalent to

$$
[d x]_{0} \notin H_{0} \oplus N\left(C^{0}\right) .
$$

This finishes the proof of our main result Theorem 2 . 


\section{REFERENCES}

[Bri70] Egbert Brieskorn, Die Monodromie der isolierten Singularitäten von Hyperflächen, Manuscripta Math. 2 (1970), 103-161. MR MR0267607 (42 \#2509)

[CJNMM96] Francisco J. Castro-Jiménez, Luis Narváez-Macarro, and David Mond, Cohomology of the complement of a free divisor, Trans. Amer. Math. Soc. 348 (1996), no. 8, 3037-3049. MR MR1363009 (96k:32072)

[CMMNMCJ02] Francisco J. Calderón Moreno, David Mond, Luis Narváez Macarro, and Francisco J. Castro Jiménez, Logarithmic cohomology of the complement of a plane curve, Comment. Math. Helv. 77 (2002), no. 1, 24-38. MR MR1898392 (2003e:32047)

[CMNM05] Francisco Javier Calderón Moreno and Luis Narváez Macarro, Dualité et comparaison sur les complexes de de Rham logarithmiques par rapport aux diviseurs libres, Ann. Inst. Fourier (Grenoble) 55 (2005), no. 1, 47-75. MR MR2141288 (2006d:32008)

[DD90] P. Deligne and A. Dimca, Filtrations de Hodge et par l'ordre du pôle pour les hypersurfaces singulières, Ann. Sci. École Norm. Sup. (4) 23 (1990), no. 4, 645656. MR MR1072821 (91m:14028)

[Del71] Pierre Deligne, Théorie de Hodge. II, Inst. Hautes Études Sci. Publ. Math. (1971), no. 40, 5-57. MR MR0498551 (58 \#16653a)

[Dim91] Alexandru Dimca, On the de Rham cohomology of a hypersurface complement, Amer. J. Math. 113 (1991), no. 4, 763-771. MR MR1118460 (93c:14021)

[GPS05] G.-M. Greuel, G. Pfister, and H. Schönemann, Singular 3.0, A Computer Algebra System for Polynomial Computations, Centre for Computer Algebra, University of Kaiserslautern, 2005, http://www.singular.uni-kl.de.

[Gri69] Phillip A. Griffiths, On the periods of certain rational integrals. I, II, Ann. of Math. (2) 90 (1969), 460-495; ibid. (2) 90 (1969), 496-541. MR MR0260733 (41 \#5357)

[Gro66] A. Grothendieck, On the de Rham cohomology of algebraic varieties, Inst. Hautes Études Sci. Publ. Math. (1966), no. 29, 95-103. MR MR0199194 (33 \#7343)

[GS06] Michel Granger and Mathias Schulze, On the formal structure of logarithmic vector fields, Compos. Math. 142 (2006), no. 3, 765-778. MR MR2231201 (2007e:32037)

[HM98] Martin P. Holland and David Mond, Logarithmic differential forms and the cohomology of the complement of a divisor, Math. Scand. 83 (1998), no. 2, 235-254. MR MR1673922 (2000c:32079)

[Kar91] Yakov Karpishpan, Pole order filtration on the cohomology of algebraic links, Compositio Math. 78 (1991), no. 2, 213-226. MR MR1104788 (92d:32051)

[Mal74] Bernard Malgrange, Intégrales asymptotiques et monodromie, Ann. Sci. École Norm. Sup. (4) 7 (1974), 405-430 (1975). MR MR0372243 (51 \#8459)

[Mil68] John Milnor, Singular points of complex hypersurfaces, Annals of Mathematics Studies, No. 61, Princeton University Press, Princeton, N.J., 1968. MR MR0239612 (39 \#969)

[NM08] Luis Narvaez-Macarro, Linearity conditions on the Jacobian ideal and logarithmic-meromorphic comparison for free divisors, arXiv math.AG (2008), no. $0804.2219 \mathrm{v} 1$.

[Pha77] Frédéric Pham, Caustiques, phase stationnaire et microfonctions, Acta Math. Vietnam. 2 (1977), no. 2, 35-101. MR MR0508047 (58 \#22648)

[Pha79] Singularités des systèmes différentiels de Gauss-Manin, Progress in Mathematics, vol. 2, Birkhäuser Boston, Mass., 1979, With contributions by Lo Kam Chan, Philippe Maisonobe and Jean-Étienne Rombaldi. MR MR553954 (81h:32015)

[Sai71] Kyoji Saito, Quasihomogene isolierte Singularitäten von Hyperflächen, Invent. Math. 14 (1971), 123-142. MR MR0294699 (45 \#3767)

[Sai80] Theory of logarithmic differential forms and logarithmic vector fields, J. Fac. Sci. Univ. Tokyo Sect. IA Math. 27 (1980), no. 2, 265-291. MR MR586450 (83h:32023) 
[Sai91] Morihiko Saito, Period mapping via Brieskorn modules, Bull. Soc. Math. France 119 (1991), no. 2, 141-171. MR MR1116843 (92h:32052)

[Sch02] Mathias Schulze, The differential structure of the Brieskorn lattice, Mathematical software (Beijing, 2002), World Sci. Publ., River Edge, NJ, 2002, pp. 136-146. MR MR1932606

[Sch04a] M. Schulze, gmssing.lib, Singular library, Centre for Computer Algebra, University of Kaiserslautern, 2004.

[Sch04b] Mathias Schulze, A normal form algorithm for the Brieskorn lattice, J. Symbolic Comput. 38 (2004), no. 4, 1207-1225. MR MR2094217 (2005g:14007)

[Seb70] Marcos Sebastiani, Preuve d'une conjecture de Brieskorn, Manuscripta Math. 2 (1970), 301-308. MR MR0267608 (42 \#2510)

[SS85] J. Scherk and J. H. M. Steenbrink, On the mixed Hodge structure on the cohomology of the Milnor fibre, Math. Ann. 271 (1985), no. 4, 641-665. MR MR790119 (87b:32014)

M. Schulze, Oklahoma State University, Department of Mathematics, Stillwater, OK 74078, United STATES

E-mail address: mschulze@math.okstate.edu 